\title{
Delayed dynamical sytem strongly coupled to a nonlinear energy sink : application to machining chatter
}

\author{
Etienne Gourc ${ }^{1, \text { a }}$, Sébastien Seguy ${ }^{1}$, Guilhem Michon ${ }^{2}$, and Alain Berlioz ${ }^{3}$ \\ 1 Université de Toulouse, INSA, ICA (Institut Clément Ader), F-31077, Toulouse, France \\ 2 Université de Toulouse, ISAE, ICA (Institut Clément Ader), F-31055, Toulouse, France \\ 3 Université de Toulouse, UPS, ICA (Institut Clément Ader), F-31062, Toulouse, France
}

\begin{abstract}
This paper investigate the different response regimes of a cutting tool on a lathe strongly coupled to a nonlinear energy sink. The equations of motion are analysed via the method of multiple scales. Condition of elimination of secular terms permit to derive equation of the slow invariant manifold (SIM) and the behavior of the system has been explained by studying the location of the fixed points of the slow flow on the SIM. Different types of responses are revealed such as periodic response and also strongly modulated response (SMR) wich are not related to the fixed points of the slow flow. Analytic results are then compared to numerical simulations.
\end{abstract}

\section{Introduction}

The surface quality of parts produced by machining operation is strongly affected by the well know regenerative chatter. The chatter instability is induced by the time delay between two consecutive workpiece revolution. By the effect of some external disturbance, the tool start damped oscillation relative to the workpiece, and the surface roughness is undulated. For the consecutive workpiece revolution, the chip thickness is modulated. This regenerative mechanism is well known and presented first by Tobias [1]. Since this work, many researcher have improved the knowledge by the stability lobe representation, see e.g. [2, $3]$. The behavior of a cutting tool on a lathe has also been studied using the method of multiple scales [4]. Various techniques for chatter suppression have been investigated. In [5], they used a variable spindle speed in milling to disturb the time delay. Another approach to reduce chatter is the use of linear tuned vibration absorbers. Recently, an analytical optimized method was presented for linear absorbers in the context of chatter [6]. These linear absorbers are successfully applied on boring process [7]. Active absorbers have been also proposed with piezoelectric tool [8]. However all these linear absorbers are limited by the small frequency bandwidth, and in practice their efficiency is not interesting for the machinist. The idea of attaching a nonlinear oscillator to a turning machine is relatively recent [9]. In recent studies, it has been demonstrated that addition of a small mass attachment with a strong nonlinear coupling (i.e. a nonlinear energy sink (NES)) to a linear oscillator can be benefit for vibration mitigation [10]. In [11], a general analytical procedure to deal with such systems is presented. The possibility to control self excitation regimes in a Van der Pol oscillator with a NES has been demonstrated in [12]. System with NES can exhibit regimes which are not related to fixed points, and cannot be explained using local analysis [13]. These regimes are related to relaxation oscillation of the slow flow and are

\footnotetext{
a e-mail: gourc@insa-toulouse.fr
}

also benefit for passive control. In this paper, the possibility of controlling regenerative chatter using a Nonlinear Energy Sink (NES) is analyzed for turning process. Theoretical predictions are confirmed with numerical integration. The paper is organized as follow. In the next section, the model considered in this study is described. In the third section, the underlying linear sub-system is analyzed. In the fourth section the asymptotic analysis of the equation of motion is performed. Then, different response regimes accompanied with numerical simulation are presented.

\section{Mechanical model}

The model studied herein consist of a lathe cutting tool with an embeded NES. Only the first flexible mode of the cutting tool is considered, and the workpiece is assumed to be rigid. A shematic of the model is given in Fig. 1 and the governing equation of motion are as follow:

$$
\begin{aligned}
m_{1} \ddot{x}+c_{1} \dot{x}+k_{1} x+c_{2}(\dot{x}-\dot{y})+k_{2}(x-y)^{3} & =F(\Delta h(t)) \\
m_{2} \ddot{y}+c_{2}(\dot{y}-\dot{x})+k_{2}(y-x)^{3} & =0
\end{aligned}
$$

Where $m_{1}, c_{1}, k_{1}$ and $m_{2}, c_{2}, k_{2}$ are the mass, damping and stiffness of the cutting tool and the NES respectivelly. $F(\Delta(h))$ is the non constant part of the cutting force expressed as:

$$
F(\Delta h(t))=a_{p} K_{t}(x(t-\tau)-x(t))
$$

Where $a_{p}$ is the depth of cut, $K_{t}$ is a cutting stiffness, $x(t)$ is the current position of the tool and $x_{\tau}=x(t-\tau)$ is the delayed position. $\tau$ is the time delay which correspond to one workpiece revolution:

$$
\tau=\frac{2 \pi}{\Omega}
$$

Where $\Omega$ is the workpiece rotating speed. The nonlinear part of the cutting force [14] is not taken into account 


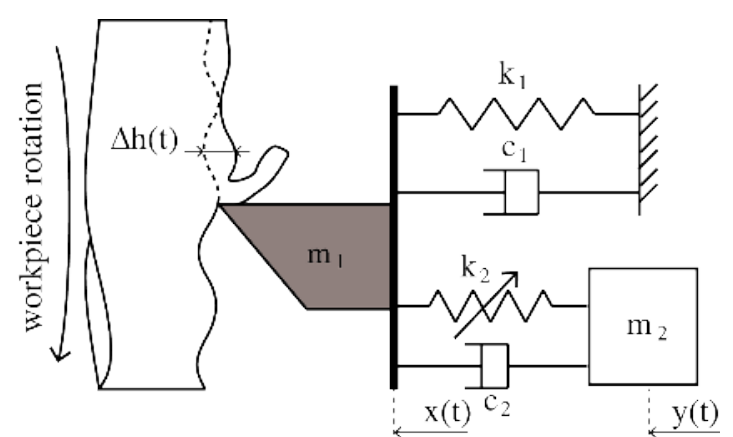

Fig. 1: Scheme of the model

in this study. After rescalling, system $(1,2)$ is rewritten in a more convenient form:

$$
\begin{gathered}
\ddot{x}+\lambda_{1} \dot{x}+x+p\left(x-x_{\tau}\right)+\varepsilon \lambda_{2}(\dot{x}-\dot{y})+\varepsilon K(x-y)^{3}=0 \\
\ddot{y}+\lambda_{2}(\dot{y}-\dot{x})+K(y-x)^{3}=0
\end{gathered}
$$

with

$$
\begin{array}{r}
\tilde{t}=\omega_{1} t, \quad \varepsilon=m 2 / m 1, \quad \omega_{1}^{2}=k_{1} / m_{1}, \quad \omega_{2}^{2}=k_{2} / m 2, \\
K=\omega_{2}^{2} / \omega_{1}^{2}, \quad \lambda_{1}=c_{1} /\left(m_{1} \omega_{1}\right), \quad \lambda_{2}=c_{2} /\left(m_{2} \omega_{1}\right), \\
p=a_{p} K_{t} /\left(m_{1} \omega_{1}^{2}\right), \quad \tilde{\tau}=\omega_{1} \tau
\end{array}
$$

\section{Analysis of the uncoupled system}

In this section, the behavior of the lathe cutting tool alone is analysed. The procedure is described more in details in [4]. The linearized equation of a cutting tool on a lathe is given by:

$$
\ddot{x}+\lambda_{1} \dot{x}+x+p\left(x-x_{\tau}\right)=0
$$

Equation (7) admit solutions of the form:

$$
x(t)=x_{0} \mathrm{e}^{(\gamma+i \omega) t}
$$

where $\omega$ is the frequency of oscillations, $\gamma$ is the grow or decay rate, and $x_{0}$ depends on the initial conditions. The value $\gamma=0$ define the stability boundary. Substituting (8) into (7), splitting into real and imaginary parts and setting $\gamma=0$ yields:

$$
\begin{gathered}
1-\omega^{2}+p-p \cos (\omega \tau)=0 \\
\lambda_{1} \omega+p \sin (\omega \tau)=0
\end{gathered}
$$

It is possible to proove that the value $\gamma=0$ correspond to a Hopf bifurcation [4]. Solving $(9,10)$ for $\cos (\omega \tau)$ and $\sin (\omega \tau)$ and using trigonometric identity, the frequency of the bifurcated periodic orbit is obtained:

$$
\omega_{c}=\frac{1}{2} \sqrt{4 \pm 2 \sqrt{-4 \lambda_{1}^{2}+4 p_{c}^{2}-4 p_{c} \lambda_{1}^{2}+\lambda_{1}^{4}}+4 p_{c}-2 \lambda_{1}^{2}}
$$

The stability boundary is often plot in parameter space $(\Omega, p)$ and is called stability lobes. An exemple is given in Fig. 2 for $\lambda_{1}=0.1$. The regions under the curves are stables, and unstables elsewhere.

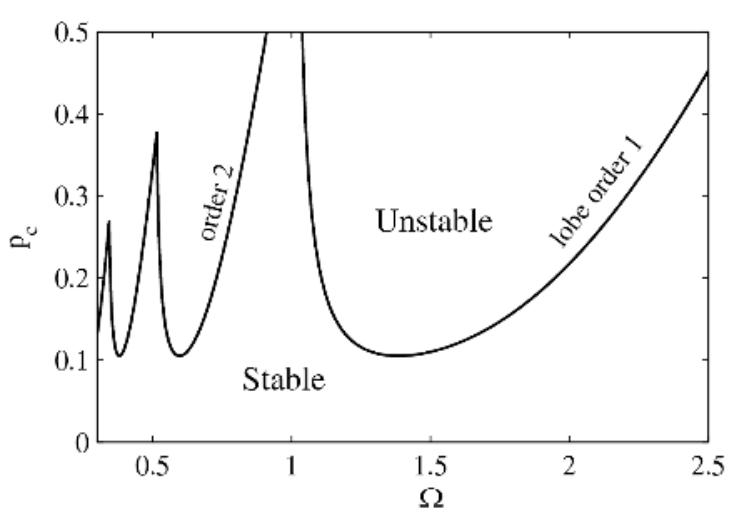

Fig. 2: Stability lobes with $\lambda_{1}=0.1$

\section{Study of the coupled system}

In this section, the coupled system is analysed. A new coordinate representing the internal displacement of the NES is introduced:

$$
w=x-y
$$

Taking into account $(12)$, system $(5,6)$ becomes:

$$
\ddot{x}+\lambda_{1} \dot{x}+x+p\left(x-x_{\tau}\right)+\varepsilon \lambda_{2} \dot{w}+\varepsilon K w^{3}=0
$$

$$
\ddot{w}+\lambda_{1} \dot{x}+x+p\left(x-x_{\tau}\right)+(1+\varepsilon) \lambda_{2} \dot{w}+(1+\varepsilon) K w^{3}=0
$$

A new small parameter $\chi$ is introduced, and the variables are rescalled as follow:

$$
\chi=\varepsilon^{1 / 3}, \quad X=\chi^{-1} x, \quad W=w
$$

Substituting (15) into $(13,14)$, and keeping only terms up to order $\chi^{2}$ yields:

$$
\begin{aligned}
& \ddot{X}+X+\lambda_{1} \dot{X}+p\left(X-X_{\tau}\right)+\chi^{2} \lambda_{2} \dot{W}+\chi^{2} K W^{3}=0 \\
& \ddot{W}+\chi X+\chi \lambda_{1} \dot{X}+\chi p\left(X-X_{\tau}\right)+\lambda_{2} \dot{W}+K W^{3}=0
\end{aligned}
$$

The system is analysed in the case of $1: 1$ resonance. In this case all variables oscillates at the same frequency, that is at the frequency of the bifurcated periodic orbit. System $(16,17)$ may be reshaped as follow:

$$
\begin{array}{r}
\ddot{X}+X+\lambda_{1} \dot{X}+p\left(X-X_{\tau}\right)+\chi^{2} \lambda_{2} \dot{W}+\chi^{2} K W^{3}=0 \\
\ddot{W}+\omega_{c} W+\chi\left[\delta\left(\lambda_{2} \dot{W}+K W^{3}-\omega_{c} W\right)+X+\lambda_{1} \dot{X}\right. \\
\left.+p\left(X-X_{\tau}\right)\right]=0
\end{array}
$$

Where $\delta=\chi^{-1}$. System $(18,19)$ is the basis for the analysis. A detuning parameter representing the nearness of $p$ to the critical value $p_{c}$ is introduced as:

$$
p=p_{c}+\chi^{2} \sigma
$$

System $(18,19)$ is analysed using the method of multiple scales. A second order uniform approximation of its solution in the vicinity of the Hopf bifurcation has the form: 


$$
\begin{array}{r}
X(t ; \chi)=X_{0}\left(T_{0}, T_{1}, T_{2}\right)+\chi X_{1}\left(T_{0}, T_{1}, T_{2}\right) \\
+\chi^{2} X_{2}\left(T_{0}, T_{1}, T_{2}\right)+\ldots \\
W(t ; \chi)=W_{0}\left(T_{0}, T_{1}, T_{2}\right)+\chi W_{1}\left(T_{0}, T_{1}, T_{2}\right) \\
+\chi^{2} W_{2}\left(T_{0}, T_{1}, T_{2}\right)+\ldots
\end{array}
$$

Where $T_{n}=\chi^{n} t, n=0,1, \ldots$. The time delay is considered as $O(1)$ since the zone which present most interest for the machinist is closeto the first hopf lobe (see Fig. 2). The delay term is expressed as:

$$
\begin{array}{r}
X(t-\tau ; \chi)=X_{0}\left(T_{0}-\tau, T_{1}-\chi \tau, T_{2}-\chi^{2} \tau\right) \\
+\chi X_{1}\left(T_{0}-\tau, T_{1}-\chi \tau, T_{2}-\chi^{2} \tau\right) \\
+\chi^{2} X_{2}\left(T_{0}-\tau, T_{1}-\chi \tau, T_{2}-\chi^{2} \tau\right)+\ldots
\end{array}
$$

Substituting Eq. $(20-23)$ into $(18,19)$ and equating coefficients of like power of $\chi$ yields:

$$
\begin{gathered}
D_{0}^{2} X_{0}+\lambda_{1} D_{0} X_{0}+X_{0}+p_{c}\left(X_{0}-X_{0 \tau}\right)=0 \\
D_{0}^{2} W_{0}+\omega_{c} W_{0}=0
\end{gathered}
$$

$$
\begin{gathered}
D_{0}^{2} X_{1}+\lambda_{1} D_{0} X_{1}+X_{0}+p_{c}\left(X_{1}-X_{1 \tau}\right) \\
=-2 D_{0} D_{1} X_{0}-\lambda_{1} D_{1} X_{0}-p_{c} \tau D_{1} X_{0 \tau}
\end{gathered}
$$

$$
\begin{array}{r}
D_{0}^{2} W_{1}+\omega_{c} W_{1}=-\delta \lambda_{2} D_{0} W_{0}-\delta K W_{0}^{3}+\delta \omega_{c} W_{0} \\
-X_{0}+p_{c}\left(X_{0 \tau}-X_{0}\right)-\lambda_{1} D_{0} X_{0}-2 D_{0} D_{1} W_{0}
\end{array}
$$

$$
\begin{array}{r}
D_{0}^{2} X_{2}+\lambda_{1} D_{0} X_{2}+X_{0}+p_{c}\left(X_{2}-X_{2 \tau}\right) \\
=-2 D_{0} D_{1} X_{1}-D_{1}^{2} X_{0}+\frac{1}{2} p_{c} \tau^{2} D_{1}^{2} X_{0 \tau}-p_{c} \tau D_{2} X_{0 \tau} \\
-p_{c} \tau D_{1} X_{1 \tau}+\sigma X_{0 \tau}-2 D_{0} D_{2} X_{0}-\lambda_{1} D_{1} X_{1} \\
-\lambda_{1} D_{2} X_{0}-\sigma X_{0}-K W_{0}^{3}-\lambda_{1} D_{0} W_{0}
\end{array}
$$

Where $X_{i \tau}=X_{i}\left(T_{0}-\tau, T_{1}, T_{2}\right)$. Only the equation governing the evolution of $X_{2}$ is shown, because it is the only one used in this study.

The general solution of Eq. (24) can be expressed as:

$$
X_{0}=A\left(T_{1}, T_{2}\right) \mathrm{e}^{i \omega_{c} T_{0}}+\sum_{n=1}^{\infty}\left[A_{n}\left(T_{1}, T_{2}\right) \mathrm{e}^{\left(\gamma_{n}-i \omega_{n}\right) T_{0}}\right]+c c
$$

Where $c c$ stand for complex conjugate, $\omega_{c}$ is the critical frequecy of the oscillations on the boundary of Hopf bifurcation and is given by $(11) .\left(\gamma_{n}-i \omega_{n}\right)$ are the remaining roots of Eq. (24). Close to the Hopf bifurcation, all the roots have negative real parts exept one which change sign at the stability boundary. After transient, all the roots decay with time, and the long time behavior at $O(1)$ is given by:

$$
\begin{aligned}
& X_{0}=A\left(T_{1}, T_{2}\right) \mathrm{e}^{i \omega_{c} T_{0}}+c c \\
& W_{0}=B\left(T_{1}, T_{2}\right) \mathrm{e}^{i \omega_{c} T_{0}}+c c
\end{aligned}
$$

Substituting $(30,31)$ into $(26,27)$ yields:

$$
\begin{array}{r}
D_{0}^{2} X_{1}+\lambda_{1} D_{0} X_{1}+X_{0}+p_{c}\left(X_{1}-X_{1 \tau}\right) \\
=\left(-2 i \omega_{c} D_{1} A-p_{c} \tau D_{1} A-\lambda_{1} D_{1} A\right) \mathrm{e}^{i \omega_{c} T_{0}}+c c \\
D_{0}^{2} W_{1}+\omega_{c} W_{1}=\left(-\delta \lambda_{2} B i \omega_{c}-3 \delta K B^{2} B^{*}\right. \\
+\delta \omega_{c} B-A+p_{c} A \mathrm{e}^{-i \omega_{c} \tau}-i \lambda_{1} \omega_{c} A-p_{c} A \\
\left.-2 i \omega_{c} D_{1} B\right) \mathrm{e}^{i \omega_{c} T_{0}}+N S T+c c
\end{array}
$$

Where NST stands for non secular terms, and the star (*) for the complex conjugate. Eliminatig terms that produce secular terms in Eq. (32), we obtain:

$$
A\left(T_{1}, T_{2}\right)=A\left(T_{2}\right)
$$

which means that $A$ does not depend on time scale $T_{1}$, therefore:

$$
X_{0}=X_{1}=A\left(T_{2}\right) \mathrm{e}^{i \omega_{c} T_{0}}+c c
$$

Now, eliminating terms that produce secular terms in (33) gives:

$$
\begin{array}{r}
-\delta \lambda_{2} i \omega_{c} B-3 \delta K B^{2} B^{*}+\delta \omega_{c} B+p_{c} A \mathrm{e}^{-i \omega_{c} \tau} \\
-A-2 i \omega_{c} D_{1} B-p_{c} A-\lambda_{1} i \omega_{c} A=0
\end{array}
$$

It is possible to proove with the help of Bendixon criterion, that solution of equation (36) must end or begin at fixed point of the equation and cannot be periodic. Consequently, looking for the fixed points of (36):

$$
\tilde{B}\left(T_{2}\right)=\lim _{T_{1} \rightarrow \infty} B\left(T_{1}, T_{2}\right)
$$

Then, we obtain:

$$
A=-\frac{\mathrm{e}^{i \omega_{c} \tau} \delta \tilde{B}\left(\lambda_{2} i \omega_{c}+3 K|\tilde{B}|^{2}-\omega_{c}\right)}{\mathrm{e}^{i \omega_{c} \tau}\left(1+p_{c}+i \lambda_{1} \omega_{c}\right)-p_{c}}
$$

The polar form is introduced as:

$$
\tilde{B}=N \mathrm{e}^{i \theta}
$$

Substituting (39) into (38), and expressing $A$ in terms of modulus yields:

$$
|A|^{2}=\delta^{2} Z\left(\lambda_{2}^{2} \omega_{c}^{2}+\omega_{c}^{2}+9 K^{2} Z^{2}-6 K Z \omega_{c}\right) \Gamma^{-1}
$$

with

$$
\begin{array}{r}
\Gamma=1+2 p_{c}+2 p_{c}^{2}+2 p_{c} \lambda_{1} \omega_{c} \sin \left(\omega_{c} \tau\right) \\
+\lambda_{1}^{2} \omega_{c}^{2}-2 p_{c}^{2} \cos \left(\omega_{c} \tau\right)-2 p_{c} \cos \left(\omega_{c} \tau\right)
\end{array}
$$

and

$$
N^{2}=Z
$$




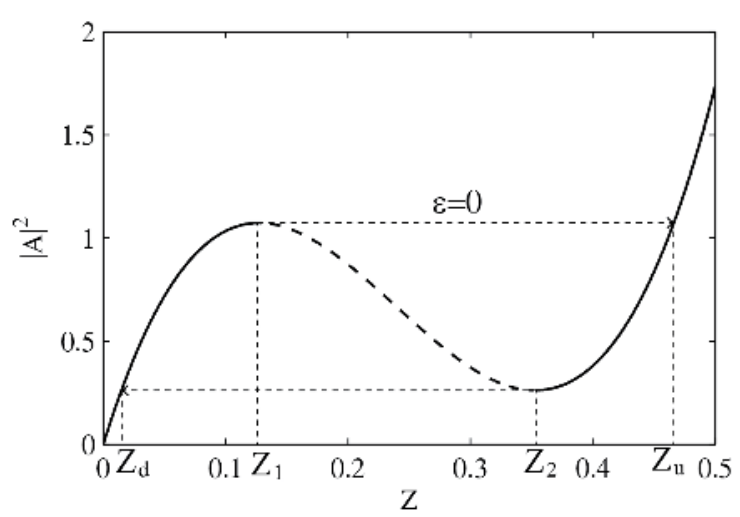

Fig. 3: Shape of the SIM in the case $\lambda_{2}<1 / \sqrt{3}$. Solid and dashed lines denotes stables and unstables branch respectivelly

Equation (40) define the slow invariant manifold (SIM) of the problem and inside its domain of attraction, variables $A$ and $B$ evolves on it. The SIM can be monotonous or have extremums. To check this possibility, the roots of the derivative of the right hand side of (40) are computed:

$$
Z_{1,2}=\frac{\left(2 \pm \sqrt{1-3 \lambda_{2}^{2}}\right) \omega_{c}}{9 K}
$$

Depending on the value of $\lambda_{2}$, the SIM can consist either of one stable branch if $\lambda_{2}>1 / \sqrt{3}$ or two stable and one unstable branch if $\lambda_{2} \leq 1 / \sqrt{3}$. In the later case, the structure of the SIM may give rise to relaxation oscillation. An exemple of such a SIM is presented in Fig. 3. In the scenario of relaxation oscillation - also called strongly modulated response $(\mathrm{SMR})$ - , the flow can achieve fold points $Z_{1}$ or $Z_{2}$, then the flow jump on the other stable branch to the landing point $Z_{u}$ or $Z_{d}$ respectively. These landing points are computed by using the invariance property of the SIM:

$$
\begin{array}{r}
\delta^{2} Z_{1,2}\left(\lambda_{2}^{2} \omega_{c}^{2}+\omega_{c}^{2}+9 K^{2} Z_{1,2}^{2}-6 K Z_{1,2} \omega_{c}\right) \\
=\delta^{2} Z_{u, d}\left(\lambda_{2}^{2} \omega_{c}^{2}+\omega_{c}^{2}+9 K^{2} Z_{u, d}^{2}-6 K Z_{u, d} \omega_{c}\right)
\end{array}
$$

then

$$
Z_{u, d}=\frac{\left(1 \pm \sqrt{1-3 \lambda_{2}^{2}}\right) 2 \omega_{c}}{9 K}
$$

To study the different response regimes on the SIM, the equation at $O\left(\chi^{2}\right)$ should be analysed. Substituting Eq. (31) and (35) into (28) yields the following equation:

$$
\begin{array}{r}
D_{0}^{2} X_{2}+\lambda_{1} D_{0} X_{2}+X_{0}+p_{c}\left(X_{2}-X_{2 \tau}\right) \\
=-p_{c} \tau D_{2} A \mathrm{e}^{-i \omega_{c} \tau}+\sigma A \mathrm{e}^{-i \omega_{c} \tau}-\lambda_{2} i \omega_{c} B-\lambda_{1} D_{2} A \\
-\sigma A-3 K B^{2} B^{*}-2 i \omega_{c} D_{2} A+N S T+c c
\end{array}
$$

Eliminating terms that produce secular terms in Eq. (46) gives:

$$
\begin{array}{r}
-p_{c} \tau D_{2} A \mathrm{e}^{-i \omega_{c} \tau}+\sigma A \mathrm{e}^{-i \omega_{c} \tau}-\lambda_{2} i \omega_{c} B-\lambda_{1} D_{2} A \\
-\sigma A-3 K B^{2} B^{*}-2 i \omega_{c} D_{2} A=0
\end{array}
$$

The equation for the SIM (40) is substituted into (47)

Splitting into real and imaginary parts, and reorganising, it is possible to obtain an expression for the derivative of $N$. The entire expression is not displayed here due to its length, but it can be expressed in more compact form as:

$$
D_{2} N=\frac{N\left(\alpha_{1} N^{4}+\alpha_{2} N^{2}+\alpha_{3}\right)}{\beta_{1} N^{4}+\beta_{2} N^{2}+\beta_{3}}
$$

Where $\alpha_{i}$ and $\beta_{i}$ are coefficients which depends only on the systems parameters. The behavior of the system can be understood by studying the fixed points of (48). From Eq. (48) it follows that a trivial fixed point is $N_{0}=0$ and the two others are expressed as:

$$
Z_{0}=\frac{-\alpha_{2} \pm \sqrt{\alpha_{2}^{2}-4 \alpha_{1} \alpha_{3}}}{2 \alpha_{1}}
$$

The different scenarii are presented in the next section.

\section{Description of some response regimes}

In this section, some different response regimes are studied. The following set of parameters has been used for each cases:

$$
\begin{array}{r}
\varepsilon=0.01, \quad \lambda_{1}=0.1, \quad \lambda_{1}=0.2, \quad K=1, \\
p_{c}=0.12, \quad \tau=3.94, \quad \omega_{c}=1.08
\end{array}
$$

Only the detunning parameter $\sigma$, which can be related to the depth of cut, will varries. Initial condition used for simulation are $x(0)=0.1$ and $\dot{x}(0)=w(0)=\dot{w}(0)=0$. The integration scheme used for numerical simulation is the Matlab dde23 algorithm. The stable fixed points on the SIM are denoted by circles, and the unstables one by cross. The NES is not optimized in this study.

\subsection{Complete suppression of chatter}

In Fig. 4a, the SIM for $\sigma=0.05$ is presented. In this case, a stable fixed point exist at the origin, and an unstable one on the second stable branch of the SIM. In this case, the flow is repelled to the origin, and chatter is fully suppressed. This scenario is confirmed by numerical integration presented in Fig. 4b.

One can see that the oscillations decrease slowly to a value close to zero. It is not presented here, but for higher initial condition (initial condition on the second stable branch of the SIM), the flow will jump down to the first stable branch of the SIM as it is illustrated by the arrows on Fig. 3, and energy pumping occurs. Numerically, we have found that such a scenario occurs for $\sigma<0.052$. For higher values of $\sigma$, other mechanism are observed. 


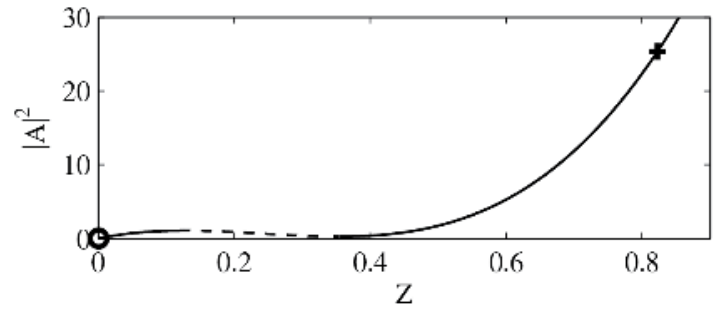

(a) Structure of the SIM. 'o' and '+' denotes stable and unstable fixed points respectively
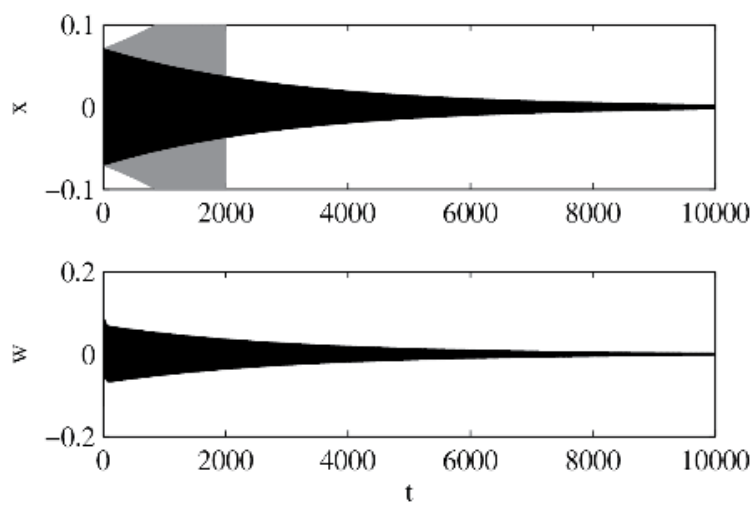

(b) Numerical verification; black line : coupled system, gray line : uncoupled system

Fig. 4: Case of complete suppression of chatter for the set of parameters (50) and $\sigma=0.05$

\subsection{Stabilisation of chatter}

In Fig. 5a, the SIM is presented for a slightly higher value of detunning parameter : $\sigma=0.09$. Now the only stable fixed point is located on the first stable branch of the SIM and correspond to small oscillation amplitude. Numerical verification is presented is Fig. 5b. The amplitude of oscillation growth slowly and stabilise at the fixed point. This senario occurs until the fixed point reach the fold point $Z_{1}$ (see Fig. 3).

\subsection{Chatter control throught SMR}

Further increase of $\sigma$, brings the possibility of appearance of SMR. As it it illustrated on the SIM in Fig. 6a for $\sigma=$ 0.2 , there exist two unstable fixed points on each stable branch of the SIM, and another unstable fixed point on the unstable branch of the SIM. In this case, the only way for the flow is to perform ralaxation cycle oscillations called SMR response. This regime is also verified numerically in Fig. 6b. It is interesting to find the value of $\sigma$ for which SMR appears. This arise when the fixed point on the first stable branch of the SIM reach the first fold point $Z_{1}$. The value of $\sigma$ where this transition occurs was $\sigma=0.120$ numerically, versus $\sigma=0.111$ analytically. This is in good agreement despite the fact that the parameter $\chi$ is not very small $(\chi=0.215)$.

\subsection{Loss of stability}

Again increasing the value of $\sigma$, the fixed point located on the second stable branch of the SIM goes down until it

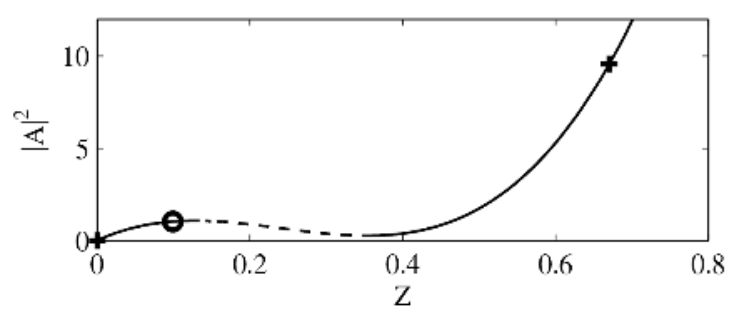

(a) Structure of the SIM. 'o' and '+' denotes stable and unstable fixed points respectively
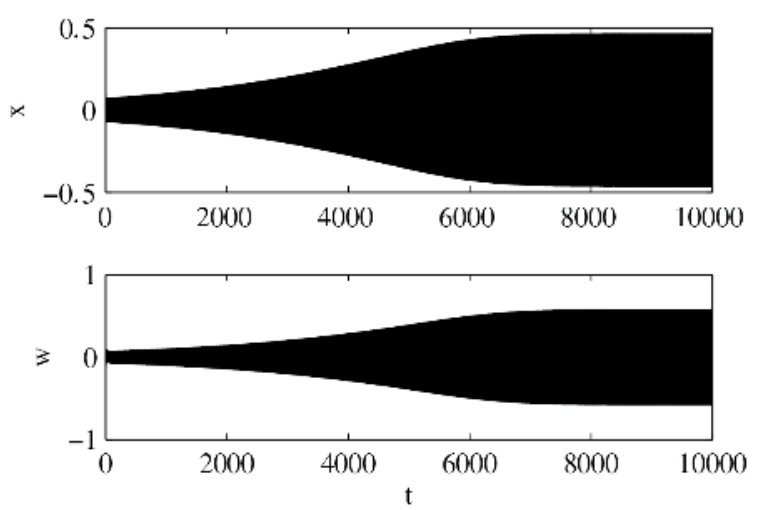

(b) Numerical verification

Fig. 5: Case of stabilization of chatter for the set of parameters $(50)$ and $\sigma=0.09$

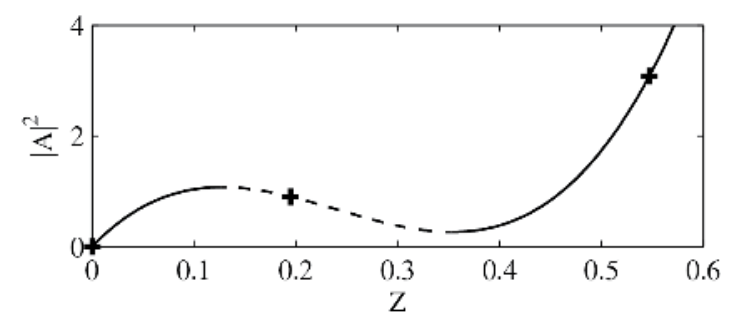

(a) Structure of the SIM. 'o' and '+' denotes stable and unstable fixed points respectively
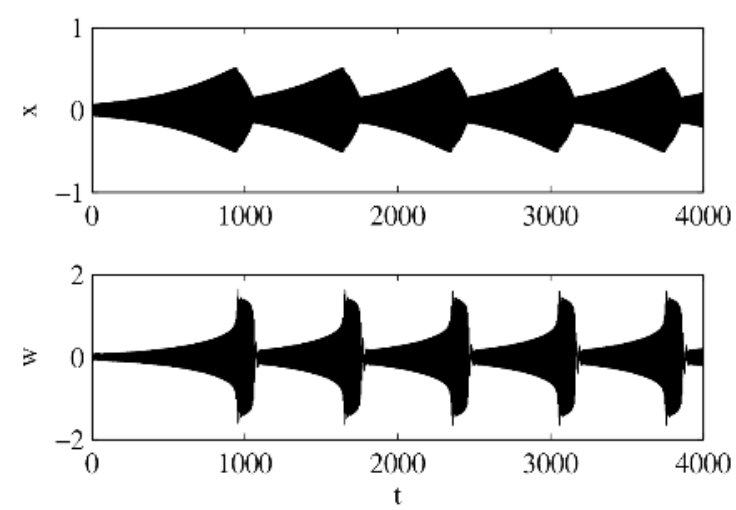

(b) Numerical verification

Fig. 6: Case of passive control of chatter via SMR for the set of parameters (50) and $\sigma=0.2$ 


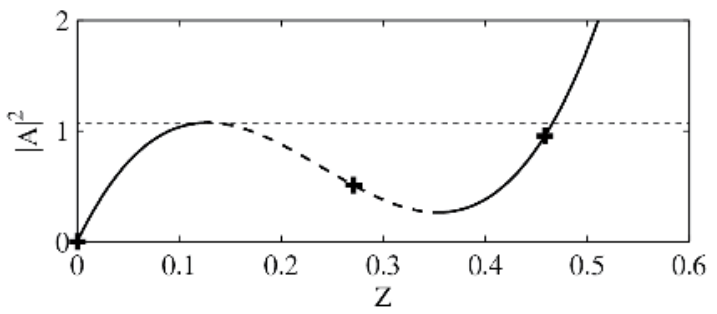

(a) Structure of the SIM. 'o' and '+' denotes stable and unstable fixed points respectively
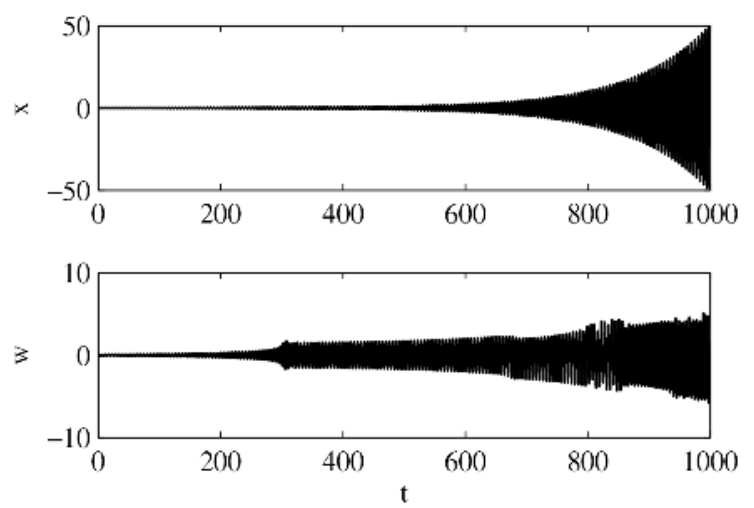

(b) Numerical verification

Fig. 7: Loss of stability for the set of parameters (50) and $\sigma=0.5$

reaches the saddle point $Z_{u}$ yielding to an homoclinic connection. A slightly increase of $\sigma$, makes system unstable. In this case, with initial conditions on the first branch of the SIM, the flow must grow on this branch until the fold point $Z_{1}$, then land on the second stable branch, and increase infinitelly (unstable). This is fully verified in Fig. 7a,7b, where the motion becomes unstable after one half SMR cylce. Numerically, the value of $\sigma$ where SMR loss stability is $\sigma=0.409$ versus $\sigma=0.455$ analytically.

\section{Concluding remarks}

In this paper, the possiblity of passivelly controling machinning chatter instability with a nonlinear energy sink was studied. The system considered consist of a linear oscillator representing a flexible lathe tool, subject to a regenerative cutting force and strongly coupled to a NES. Only the linear part of the cutting force was considered. The whole system has been studied using the method of multiple scales. Different responses regimes were revealed by studying the location of the fixed points on the SIM. Qualitativelly, the comparison between analytical prediction and numerical simulation is very satisfying. Quantitatively, some discrepancies arise in the determination of the critical parameters, certainely due to the not so small value of $\chi$. The potential benefit of the NES to control machining chatter has been demonstrated. It should be interesting to include a non linear cutting law, and to study the system behavior for a larger set of parameters and an optimized NES in a further study.

\section{References}

1. S.A. Tobias and W. Fishwick. Theory of regenerative machine tool chatter. Engineer, 205:199-203, 1958.

2. B.P. Mann, T. Insperger, G. Stépán, and P.V. Bayly. Stability of up-milling and down-milling, part 2 : experimental verification. International Journal of Machine Tools and Manufacture, 43:35-40, 2003.

3. E. Gourc, S. Seguy, and L. Arnaud. Chatter milling modeling of active magnetic bearing spindle in highspeed domain. International Journal of Machine Tools and Manufacture, 51:928-936, 2011.

4. A.H. Nayfeh and N.A. Nayfeh. Analysis of the cutting tool on a lathe. Nonlinear Dynamics, 63:395-416, 2010.

5. S. Seguy, T. Insperger, L. Arnaud, G. Dessein, and G. Peigné. Suppression of period doubling chatter in high-speed milling by spindle speed variation. $\mathrm{Ma}$ chining Science and Technology, 15:153-171, 2011.

6. N.D. Sims. Vibration absorbers for chatter suppression: a new analytical tuning methodology. Journal of Sound and Vibration, 301:592-607, 2007.

7. H. Moradi, F. Bakhtiari-Nejad, and M.R. Movahhedy. Tuneable vibration absorber design to suppress vibrations: an application in boring manufacturing process. Journal of Sound and Vibration, 318:93-108, 2008.

8. A. Harms, B. Denkena, and N. Lhermet. Tool adaptator for active vibration control in turning operations. In 9th International Conference on New Actuators, Brême, Germany, 2004.

9. M. Wang. Feasibility study of nonlinear tuned mass damper for machining chatter suppression. Journal of Sound and Vibration, 330:1917-1930, 2011.

10. O.V. Gendelman, E. Gourdon, and C.H. Lamarque. Quasiperiodic energy pumping in coupled oscillators under periodic forcing. Journal of Sound and Vibration, 294(4-5):651-662, 2006.

11. O.V. Gendelman. Bifurcations of nonlinear normal modes of linear oscillator with strongly nonlinear damped attachment. Nonlinear Dynamics, 37:115128, 2004.

12. O.V. Gendelman and T. Bar. Bifurcations of selfexcitation regimes in a van der pol oscillator with a nonlinear energy sink. Physica D: Nonlinear Phenomena, 239(3-4):220-229, 2010.

13. Y. Starosvetsky and O.V. Gendelman. Strongly modulated response in forced 2dof oscillatory system with essential mass and potential asymmetry. Physica D: Nonlinear Phenomena, 237(13):1719-1733, 2008.

14. T. Kalmár-Nagy, G. Stépán, and F.C. Moon. Subcritical hopf bifurcation in the delay equation model for machine tool vibrations. Nonlinear Dynamics, 26:121-142, 2001 . 